\title{
Mitigating the Impact of High-Speed Craft
}

\author{
Dr. Rob Langlois \\ Department of Mechanical and Aerospace Engineering \\ Carleton University
}

High-speed craft (HSC) are relatively small maritime vessels that can operate at high speed in up to moderate sea conditions. Due to their versatility and performance they are used extensively for military operations, law enforcement, and tourism. The nature of the boat design and operation often exposes occupants to a repeated shock environment that leads to both acute and chronic neck and spinal injuries while also contributing to the immediate effects of reduced physical and cognitive performance. To mitigate these adverse outcomes, the industries that employ these boats have been rapidly transitioning from fixed seating to sophisticated suspension seating. The resulting demand has led to a wide variety of suspension seat designs becoming commercially available and widely adopted. To some extent, seat design has outpaced the systematic development of design requirements and the ability to objectively compare the relative performance of seats in consistent, reproducible, and representative conditions. This presentation will introduce the topic of HSC, discuss applied dynamics challenges associated with shock mitigation seat evaluation, and overview recent research and development work that has been on-going in the Carleton University Applied Dynamics Laboratory related to HSC seat testing. 